\title{
LINEAR AND CUBIC DYNAMIC SUSCEPTIBILITIES IN QUANTUM SPIN GLASS
}

\author{
G. Busiello ${ }^{1}$, R. V. Saburova ${ }^{2}$, V. G. Sushkova ${ }^{2}$ \\ ${ }^{1}$ Dipartimento di Fisica "E.R. Caianiello", Università di Salerno, \\ 84081 Baronissi-Salerno and INFM - Unità di Salerno, Salerno, Italy. \\ ${ }^{2}$ Department of Physics, Kazan State Power-Engineering University, \\ Krasnoselskaya Str. 51, Kazan, 420066 Russia.
}

(Received August 30, 2001)

\begin{abstract}
The low temperature behaviour of the dynamic nonlinear (cubic) susceptibility $\chi_{3}^{\prime}(\omega, T)$ in quantum $d$-dimensional Ising spin glass with short-range interactions between spins is investigated in terms of the quantum droplet model and the quantum-mechanical nonlinear response theory is employed. We have revealed a glassy like behaviour of droplet dynamics. The frequency dependence of $\chi_{3}^{\prime}(\omega, T)$ is very remarkable, the temperature dependence is found at very low temperatures (quantum regime). The nonlinear response depends on the tunneling rate for a droplet which regulates the strength of quantum fluctuations. This response has a strong dependence on the distribution of droplet free energies and on the droplet length scale average. Implications for experiments in quantum spin glasses like disordered dipolar quantum Ising magnet $\mathrm{LiHo}_{x} \mathrm{Y}_{1-x} \mathrm{~F}_{4}$ and pseudospin are noted.
\end{abstract}

Key words: spin glasses, phase transition

PACS number(s): 75.40.Gb, 75.10.Nr, 64.70.Pf

\section{INTRODUCTION}

The dynamics of glassy systems is an attractive and rapidly developing field of physics [1-5]. Spin glasses and quantum spin glasses are a very interesting system for a theoretical as well as an experimental investigation of dynamic phenomena [2-4]. There are two different spin glass dynamic descriptions: the mean-field theory and the droplet phenomenological one [1-6]. In this paper we investigate theoretically nonlinear cubic dynamic susceptibility as a function of frequency and temperature in the Ising spin glass in a transverse field in terms of quantum droplet model at very low temperatures (quantum regime). The quantum phase transitions which are governed by quantum fluctuations of the system may tunnel from one local minimum of the free energy to another; new physical effects such as quantum channel of relaxation appear. There are few theoretical studies on the nonlinear static response in quantum spin glasses [7-10] and almost no studies on the dynamic nonlinear response [3]. In [3] the dynamic nonlinear response of a quantum spin glass was found to be frequency independent and nonsingular in quantum critical regime in contrast to its behaviour in the usual spin glass. There are experimental data on the nonlinear dynamic response in classical [11-13] and quantum [14] spin glasses investigated by the Fourier-transform technique. A third-order nonlinear susceptibility is negative and diverges at an ordinary spin glass transition temperature $T_{g}$ from both the upper and the lower sides. But when $\chi_{3}^{\prime}$ is measured by a finite probing frequency the response falls out of equilibrium before the transition temperature and does not diverge at $T_{g}$. Then $\chi_{3}^{\prime}(\omega)$ shows a maximum at $T \simeq T_{f}(\omega)$ where $T_{f}(\omega)$ is the freezing temperature which is the upper bound on $T_{g}$ and $T_{g}=T_{f}(\omega \rightarrow 0)$. Such a behaviour was observed, for the example, for classical Ising spin glass $\mathrm{Fe}_{0.5} \mathrm{Mr}_{0.5} \mathrm{TiO}_{3}[13]$. W. Wu et al.
[14] measured nonlinear susceptibility $\chi_{3}^{\prime}(\omega, T)$ in quantum spin glass (the diluted dipolar-coupled Ising spin glass $\mathrm{LiHo}_{0.167} \mathrm{Y}_{0.833} \mathrm{~F}_{4}$ in the transverse field) tuning transverse field $\Gamma$ from the $\Gamma=0$ classical to the $T=0$ quantum limit. At the $\mathrm{mK}$ temperatures they found a clear dynamic signature of the spin glass to paramagnet transition whether dominated by thermal or quantum fluctuations. In [14] it was shown that $\chi_{3}^{\prime}$ depends on the frequency for $\omega>10 \mathrm{~Hz}$. However, it depends very weakly on $\omega$ for $\omega<10 \mathrm{~Hz}$. There is a crossover between high $\omega$ ( $\omega$-dependent) and low $\omega$ ( $\omega$-independent) behaviours. Nonlinear susceptibility contains a diverging component which dominates at $T=98 \mathrm{mK}$, but disappears by $25 \mathrm{mK}$. The $\chi_{3}^{\prime}(\omega)$ does not diverge but shows a maximum at $T_{f}(\omega)$. $\chi_{3}^{\prime}(\omega)$ measured at a higher temperature and lower transverse field has a larger maximum than the $\chi_{3}^{\prime}(\omega)$ measured at a lower temperature and a larger transverse field. The analysis of these experimental data seems not clear [15] because frequencies used in the experiments [14] are not sufficiently low such as to determine the equilibrium behaviour of system. Contrary to the theoretical expectations, quantum transitions may be qualitatively different from thermally driven transitions in real spin glasses. Recently the linear dynamic susceptibility (in-phase and out of phase components) at $T=0$ was investigated theoretically for the Ising spin glass in a transverse field in terms of the quantum droplet model by M. J. Thill and D. A. Huse [6]. At present two different dynamic descriptions exist: the mean field theory and the droplet picture (more phenomenological but more real). It is better to understand the actual argument of discussion [5]. A basic assumtion of the droplet picture is that the spin glass dynamics is governed by large-scale excitations whose relaxation time increases with length scale. There we give an employment of droplet spin glass model to a dynamic nonlinear response at very low temperatures. In previous papers [16] we have calculated the 
real and imaginary parts of linear dynamic susceptibility in the same model, as in [6], for nonzero temperatures. The temperature and frequency dependence of susceptibilities $\chi_{1}^{\prime}(\omega, T)$ and $\chi_{1}^{\prime \prime}(\omega ; T)$ is calculated at low temperatures. We find a broad maximum for the real part $\chi_{1}^{\prime}(\omega, T)$ which shifts towards higher temperatures with increasing frequency of ac external field $\omega$. The temperature of maximum $T_{f}(\omega)$ depends on frequency $\omega$ and increases with $\omega$ up to the magnitude where $\chi_{1}^{\prime}(\omega, T)$ starts to decrease. The imaginary part $\chi_{1}^{\prime \prime}(\omega, T)$ has a very interesting behaviour too. It is shown that many low temperature properties are dominated by thermally active droplets at the classical-to-quantum crossover length scale of droplets. In contrast to the linear susceptibility, the nonlinear dynamic cubic susceptibility diverges or converges depending on the kind of distribution of free droplet energies. It is shown that both susceptibilities have a glassy behaviour. In [16] the general linear response theory of magnetic dispersion and absorption phenomena for quantum systems by Kubo and Tomita [17] that relates the relaxation function and the linear dynamic susceptibility was used. But for simplicity dipoledipole and dipole-lattice interactions were neglected. We note that in [6] the real part of the cubic nonlinear ac susceptibility was defined as the in-phase $3 \omega$ magnetization response $M(3 \omega)$ to a small time-dependent applied field $h \cos (\omega t)$ :

$$
\chi_{3}^{\prime}=\lim _{h \rightarrow 0} \frac{24 M(3 \omega)}{h^{3} V},
$$

where $V$ is the sample volume. The authors of [6] gave some expression for $\chi_{3}^{\prime}$ they expect only at zero temperature.

\section{DYNAMICAL NONLINEAR RESPONSE}

The full nonlinear response theory despite its generality and importance is of limited practical value because it is mathematically difficult. Eventually it is necessary to make approximations, for example, the wellknown perturbation expansion of the time-evolution operators, using perturbation Hamiltonian with some small parameter. Nonlinear response theory was developed and described, for example, in [17-22]. Here we summarize briefly the theory of higher-order dynamic response. It is based on the Hamiltonian

$$
\hat{\mathcal{H}}_{t}=\hat{\mathcal{H}}_{0}+\hat{\mathcal{H}}_{1}=\hat{\mathcal{H}}_{0}-\hat{\mathcal{A}}_{j} F_{j}(t), t \geq t_{0}
$$

where $\hat{\mathcal{H}}_{0}$ is nonperturbation Hamiltonian of system, $\hat{\mathcal{H}}_{1}$ is perturbation Hamiltonian which describes the interaction between the Heisenberg operators $\hat{\mathcal{A}}_{j}$ (material operators) and the external perturbation $\hat{\mathcal{F}}_{j}$. At time $t>t_{0}$ one is interested in the expectation value of the Heisenberg operator $\hat{\mathcal{B}}_{i}$ which is given by

$$
\left\langle\hat{B}_{i}(t)\right\rangle=\operatorname{Tr}\left[\rho_{0} \hat{B}_{i}(t)\right]=\operatorname{Tr}\left[\hat{\rho}(t) \hat{B}_{i}\right]
$$

where the density matrix $\hat{\rho}(t)=\hat{U}\left(t, t_{0}\right) \hat{\rho}\left(t_{0}\right) \hat{U}^{\dagger}\left(t, t_{0}\right)$. The time-evolution operator $\hat{U}$ satisfies the Schrödinger equation $i \hbar(d \hat{U} / d t)=\hat{\mathcal{H}} \hat{U}$. This differential equation is equivalent to an integral equation which has the interactive solution of the form [18-20]

$$
\begin{aligned}
& \hat{U}\left(t, t_{0}\right)=T \exp \left[-\frac{i}{\hbar} \int_{t_{0}}^{t} \mathcal{H}\left(t^{\prime}\right) d t^{\prime}\right] \simeq \\
& \hat{U}_{0}\left(t, t_{0}\right)\left\{1+\frac{i}{\hbar} \int_{t_{0}}^{t} d t_{1} F_{j}\left(t_{1}\right) \hat{U}_{0}^{\dagger}\left(t_{1}, t_{0}\right) \hat{A}_{j} \hat{U}_{0}\left(t_{1}, t_{0}\right)\right\},
\end{aligned}
$$

where $\hat{U}_{0}\left(t, t_{0}\right)=\exp \left[-\frac{i}{\hbar}\left(t-t_{0}\right) \hat{\mathcal{H}}\right]$. It is difficult to find an expression for $\hat{U}$ in closed form. It was used that $\hat{\mathcal{H}}_{1}(t)$ is in some sense small. We define the total response of the system at time $t$ to the external force $F_{j}$ as the difference

$$
\Delta B_{i}(t) \equiv\left\langle\hat{B}_{i}(t)\right\rangle-\left\langle\hat{B}_{i}\right\rangle_{0}
$$

where the subscript zero on expectation values refers to the equilibrium expectations. One can then understand the behaviour of the system in terms of the dynamical response. Using aforementioned expressions [2-4] the dynamical response can be written through the third order in the perturbation $\hat{\mathcal{H}}_{1}$ in the following form [20]

$$
\begin{aligned}
& \left\langle\hat{B}_{i}(t)\right\rangle-\left\langle\hat{B}_{i}\right\rangle_{0}=\Delta B_{i}(t) \simeq \int_{t_{0}}^{t} d t^{\prime} \varphi_{i j}\left(t-t^{\prime}\right) F_{j}\left(t^{\prime}\right) \\
& +\int_{t_{0}}^{t} d t_{1} \int_{t_{0}}^{t_{1}} d t_{2} \varphi_{i j k}\left(t-t_{2}, t_{1}-t_{2}\right) F_{k}\left(t_{1}\right) F_{j}\left(t_{2}\right) \\
& +\int_{t_{0}}^{t} d t_{1} \int_{t_{0}}^{t_{1}} d t_{2} \int_{t_{0}}^{t_{2}} d t_{3} \varphi_{i j k l}\left(t-t_{3}, t_{2}-t_{3}, t_{1}-t_{2}\right) \\
& \times F_{e}\left(t_{1}\right) F_{k}\left(t_{2}\right) F_{j}\left(t_{3}\right)+\ldots,
\end{aligned}
$$

where $\varphi_{i j}, \varphi_{i j k}, \varphi_{i j k l}$ are the first-, second- and third order response functions,

$$
\begin{aligned}
& \varphi_{i j}\left(t-t^{\prime}\right)=\frac{1}{i \hbar}\left\langle\left[\hat{A}_{j}, B_{i}\left(t-t^{\prime}\right)\right]\right\rangle_{0}, \\
& \varphi_{i j k}\left(t-t_{2}, t_{1}-t_{2}\right)=\frac{1}{(i \hbar)^{2}} \\
& \times\left\langle\left[\hat{A}_{j},\left[\hat{A}_{k}\left(t_{1}-t_{2}\right), \hat{B}_{i}\left(t-t_{2}\right)\right]\right]\right\rangle_{0}, \\
& \varphi_{i j k l}\left(t-t_{3}, t_{2}-t_{3}, t_{1}-t_{2}\right)=\frac{1}{(i \hbar)^{3}} \\
& \times\left\langle\left[\hat{A}_{j},\left[A_{k}\left(t_{1}-t_{2}\right),\left[A_{l}\left(t_{2}-t_{3}\right), B_{i}\left(t-t_{3}\right)\right]\right]\right]\right\rangle_{0} .
\end{aligned}
$$


Here we have employed the summation convention over repeated indices and cyclic invariance of the trace. Expressions [7-9] can be written in a more revealing form if we set $t_{0}=-\infty$ and change integration variables including an adiabatic switching factor if necessary. We take (instead of $\left.t, t_{1}, t_{2}, t_{3}\right) \tau_{1}=t-t_{1}, \tau_{2}=t_{1}-t_{2}$ time differences further. The ordinary linear response theory utilized only $\varphi_{i j}\left(\tau_{1}\right)$ and it is the simplest approximation to the full theory of linear dynamic response [23]. Using new variables we may write the expressions for response functions in the form

$$
\begin{aligned}
& \varphi_{i j}^{(1)}=-\frac{1}{i \hbar}\langle[A(\tau), B(0)]\rangle, \\
& \varphi_{i j k}^{(2)}=\frac{1}{(i \hbar)^{2}}\left\langle\left[\left[A\left(\tau_{1}+\tau_{2}\right), A\left(\tau_{2}\right)\right], B\right]\right\rangle, \\
& \varphi_{i j k l}^{(3)}=-\frac{1}{(i \hbar)^{3}}\left\langle\left[\left[\left[ A\left(\tau_{1}+\tau_{2}+\tau_{3}\right),\right.\right.\right.\right. \\
& \left.\left.\left.\left.A\left(\tau_{2}+\tau_{3}\right)\right], A\left(\tau_{3}\right)\right], B\right]\right\rangle,
\end{aligned}
$$

where the bracket $\langle\ldots\rangle$ denotes an expectation value with respect to the equilibrium ensemble. The useful interpretation is generated from Eq. [6] in the case that $t_{0}=-\infty$ if we suppose that the external force $F$ is constant and vanishes for $t \geq 0$. For $t=0$ the system is in partial equilibrium and starts to relax to equilibrium. It is convenient to write a nonlinear response for this case (initial value case [21]) as

$$
\begin{aligned}
& \langle\hat{B}(t)\rangle-\langle\hat{B}\rangle_{0}=R^{(1)}(t) F \\
& +\frac{1}{2} R^{(2)}(t) F F+\frac{1}{3} R^{(3)}(t) F F F+\ldots,
\end{aligned}
$$

where $R^{\alpha}(t)$ are the relaxation functions,

$$
\begin{aligned}
& R^{(1)}(t)=\int_{0}^{\infty} d \tau \varphi_{i j}^{(1)}\left(\tau_{1}\right) \\
& R^{(2)}(t)=\int_{0}^{\infty} d \tau_{1} \int_{0}^{\infty} d \tau_{2} \varphi_{i j k}^{(2)}\left(\tau_{2}, \tau_{1}+\tau_{2}\right) \\
& R^{(3)}(t)=\int_{0}^{\infty} d \tau_{1} \int_{0}^{\infty} d \tau_{2} \\
& \times \int_{0}^{\infty} d \tau_{3} \varphi_{i j k l}^{(3)}\left(\tau_{3}, \tau_{2}+\tau_{3}, \tau_{1}+\tau_{2}+\tau_{3}\right) .
\end{aligned}
$$

In this form response may describe relaxation of the system. If response function $\varphi(t)^{(1)}$ vanishes as $t \rightarrow \infty$, then $\varphi(t)^{(1)}=-\delta R^{(1)}(t) / \delta t$, so $R^{(1)}(t)$ contains much more information than the response function.

\section{DYNAMIC MAGNETIC SUSCEPTIBILITIES}

Let the ac magnetic field $h_{\omega}=h \cos (\omega t)$ be applied to a magnetic system. The magnetization nonlinear response

$$
M(\omega, t)=\sum_{k=1}^{n}\left\{\Theta_{k}^{\prime} \cos (k \omega t)+\Theta_{k}^{\prime \prime} \sin (k \omega t)\right\}
$$

to harmonic magnetic field $h$ contains only odd harmonic: $\Theta_{1}^{\prime} \sim \chi_{1}^{\prime} h, \Theta_{3}^{\prime} \sim \chi_{3}^{\prime} h^{3}$, etc. [11]. In the expression for $M(\omega, t)$ the magnitudes $\Theta_{k}^{\prime}$ and $\Theta_{k}^{\prime \prime}$ are real and imaginary parts of harmonic amplitudes respectively. For the general theory of nonlinear processes one can evaluate [11]

$$
\begin{aligned}
\Theta_{1}^{\prime} & =\chi_{1}^{\prime}(\omega t) h+\left[\chi_{3}^{\prime}(\omega, 0, \omega)\right] \frac{h^{3}}{4} \\
& +\left[4 \chi_{5}^{\prime}(\omega, 0, \omega, 0, \omega)+2 \chi_{5}^{\prime}(\omega, 0, \omega, 2 \omega, \omega)\right. \\
& +2 \chi_{5}^{\prime}(\omega, 2 \omega, \omega, 0, \omega)+\chi_{5}^{\prime}(\omega, 2 \omega, \omega, 2 \omega, \omega) \\
& \left.+\chi_{5}^{\prime}(\omega, 2 \omega, 3 \omega, 2 \omega, \omega)\right] \frac{h^{5}}{16}+\ldots, \\
\Theta_{3}^{\prime} & =\chi_{3}^{\prime}(3 \omega, 2 \omega, \omega) \frac{h^{3}}{4}+\left[\chi_{5}^{\prime}(3 \omega, 2 \omega, 3 \omega, 2 \omega, \omega)\right. \\
& +\chi_{5}^{\prime}(3 \omega, 4 \omega, 3 \omega, 2 \omega, \omega)+2 \chi_{5}^{\prime}(3 \omega, 2 \omega, \omega, 0, \omega) \\
& \left.+\chi_{5}^{\prime}(3 \omega, 2 \omega, \omega, 2 \omega, \omega)\right] \frac{h^{5}}{16}+\ldots, \\
\Theta_{5}^{\prime} & =\chi_{5}^{\prime}(5 \omega, 4 \omega, 3 \omega, 2 \omega, \omega) \frac{h^{5}}{16}+\ldots
\end{aligned}
$$

The measurement of all the harmonic amplitudes $\Theta_{k}$ gives a measurement of the susceptibilities $\chi_{a}$ in two limits: a) if $\chi_{1}^{\prime} h \gg \chi_{3}^{\prime} h^{3} \gg \chi_{5}^{\prime} h^{5}$ the back reaction is negligible and each harmonic measures the susceptibility of the same order; b) in the static $(\omega \rightarrow 0)$ limit the solution of the linear system [16] fully accounts for the back reaction. In the absence of ac magnetic field, the back reaction can be made small so that the dynamic susceptibilities can be obtained from Eq. [16]. In a more compact notation we may write

$$
M(\omega, t) \sim\left[M_{0}+M_{\omega}+M_{3 \omega}+\ldots\right]+[\mathrm{C} . \mathrm{C} .],
$$

where $M_{0}$ is the equilibrium magnetization in zero field; $M_{\omega}$ is the $\omega$-magnetization response; $M_{3 \omega}$ is the $3 \omega$ magnetization response and so on.

The expression (10)-(12) may be considered as solution of the corresponding quantum equations considered above. For the external ac field we assume a classical value. This field interacts with quantum system and system behaviour is determined by quantum laws. We shall focus on the real part of the third-order nonlinear dy- 
namic susceptibility $\chi_{3}^{\prime}(3 \omega, 2 \omega, \omega)$ and denote it as $\chi_{3}^{\prime}(\omega)$. In this paper we are interested in the response when the ac magnetic field is applied in $z$-direction; $\chi_{1}=\chi_{z z}$ and so on. In formulas (2-12) both $A_{i}$ and $B_{i}$ are the magnetic dipole moment operators. Considering the initial value case [21] we suppose, like [6], that the system is in equilibrium with a small time-independent field $h=h_{z}$ for $t \leq 0$ and that the external field is turned off at $t=0$, then for $t \geq 0$ the induced magnetization of the sample in $z$-direction to first order of perturbation theory is given by $M(t)-M_{0} \approx R^{(1)}(t) h$, where a relaxation function

$$
R^{(1)}(t)=\int_{0}^{\infty} \varphi^{(1)}(\tau) d \tau
$$

and the first order response function is [20]

$$
\varphi_{i j}^{(1)}(\tau) \approx-\frac{1}{i \hbar}\left\langle\left[M_{i}(\tau), M_{j}\right]\right\rangle
$$

The higher-order response functions are given by

$\varphi_{i j k}^{(2)}\left(\tau_{1}, \tau_{2}\right) \approx \frac{1}{(i \hbar)^{2}}\left\langle\left[\left[M_{i}\left(\tau_{1}+\tau_{2}\right), M_{j}\left(\tau_{2}\right)\right], M_{k}\right]\right\rangle$,

$\varphi_{i j k l}^{(3)}\left(\tau_{1}, \tau_{2}, \tau_{3}\right) \approx-\frac{1}{(i \hbar)^{3}}$

$\times\left\langle\left[\left[\left[M_{i}\left(\tau_{1}+\tau_{2}+\tau_{3}\right), M_{j}\left(\tau_{2}+\tau_{3}\right)\right], M_{k}\left(\tau_{3}\right)\right], M_{l}\right]\right\rangle$.

The linear and the nonlinear dynamic susceptibilities (admittances in the spectral representation [22]) may be found through response functions (22)-(24). In order to find the complete expression for susceptibility, we should use its symmetry and causality properties [22].

The nonlinear susceptibilities may be chosen so that these susceptibilities were symmetrical relative to simultaneous permutation of tensor indices and corresponding to them arguments, for example, the second rank tensors are $\chi_{i j k}\left(\omega_{1}, \omega_{2}\right)=\chi_{i k j}\left(\omega_{2}, \omega_{1}\right)$, and the fourth rank tensors are

$$
\begin{aligned}
& \chi_{i j k l}\left(\omega_{1}, \omega_{2}, \omega_{3}\right)=\chi_{i k j l}\left(\omega_{2}, \omega_{1}, \omega_{3}\right) \\
& =\chi_{i j l k}\left(\omega_{1}, \omega_{3}, \omega_{2}\right)=\ldots
\end{aligned}
$$

according to the causality property

$$
\chi_{i j}=0 \text { for } \tau_{1}<\max \left(\tau_{2}, \tau_{3}, \ldots\right)
$$

Linear and nonlinear dynamic susceptibilities are given by

$$
\begin{aligned}
& \chi_{i j}(\omega)=\int_{0}^{\infty} d \tau \varphi_{i j}^{(1)}(\tau) e^{i \omega \tau} \\
& \chi_{i j k}\left(\omega_{1}, \omega_{2}\right)=\frac{1}{2 !} \int_{0}^{\infty} d \tau_{1}
\end{aligned}
$$

$$
\begin{aligned}
& \times \int_{0}^{\infty} d \tau_{2}\left\{\varphi_{i j k}^{(2)}\left(\tau_{1}, \tau_{2}\right) e^{i\left(\omega_{1}+\omega_{2}\right) \tau_{1}+i \omega_{1} \tau_{1}}\right\} \\
& \chi_{i j k l}\left(\omega_{1}, \omega_{2}, \omega_{3}\right)=\frac{P_{3}}{3 !} \int_{0}^{\infty} d \tau_{1} \int_{0}^{\infty} d \tau_{2} \\
& \times \int_{0}^{\infty} d \tau_{3} \varphi_{i j k l}^{(3)}\left(\tau_{1}, \tau_{2}, \tau_{3}\right) \\
& \times \exp \left[i\left(\omega_{1}+\omega_{2}+\omega_{3}\right) \tau_{1}+i \omega_{2} \tau_{2}+i \omega_{3} \tau_{3}\right] .
\end{aligned}
$$

In Eq. (28) $P_{3}$ means the summation over all permutations of the subscripts $\left(\omega_{1} j\right),\left(\omega_{2} k\right)$ and $\left(\omega_{3} l\right)$ [22]; response functions $\varphi$ are given by expressions $(22)-(24)$.

In particular, the second harmonics generation process is characterized by the tensor $\chi_{i j k}(\omega, \omega)$. If $\omega_{1}=\omega_{2}=$ $\omega_{3}=\omega$, the triple frequency $3 \omega$ is formed; the frequency tripling is described by the tensor $\chi_{i j k l}(\omega, \omega, \omega)$ (we note it as $\left.\chi_{3}^{\prime}(\omega)\right)$.

\section{THE MODEL HAMILTONIAN}

The droplet model describing the low-dimensional short-range Ising spin glass is based on renormalization group arguments [4]. In the dimensions above the lower critical dimension $d_{l}$ (usually in spin glass $2<d_{l}<3$ ) the droplet model finds a low temperature spin-glass phase in zero magnetic field. This phase differs essentially from the spin-glass phase in the mean-field approximation of the Sherrington-Kirkpatrick infinite-range spin-glass model [2]. In the droplet model there are only two pure thermodynamical states related to each other by a global spin flip. In magnetic field there is no phase transition. A droplet is an excited cluster in an ordered state where all the spins are inverted. The natural scaling ansatz for droplet free energy $\epsilon_{L}$ which are considered to be independent random variables) is $\epsilon_{L} \sim L^{\theta}, L \geq \zeta(T) ; \zeta$ is the correlation length, $L$ is the length scale of droplet and $\theta$ is the zero temperature thermal exponent, $\theta \leq(d-1) / 2$. One droplet consists of order $L^{d}$ spins. Below $d_{l}, \theta<0$; above $d_{l}$ one has $\theta>0$.

The droplet model of classical Ising spin glass was considered by D. S. Fisher and D. A. Huse [4]. The features of this model are described also, for example, in [23].

Recently M. J. Thill and D. A. Huse [6] have shown that the $d$-dimensional quantum Ising spin glass in a transverse field with the Hamiltonian

$$
\mathcal{H}=-\sum_{i, j} \mathcal{I}_{i j} S_{i}^{z} S_{j}^{z}-\Gamma \sum_{i} S_{i}^{x}
$$

( $S_{i}$ are the Pauli matrices, $\Gamma$ is the strength of the transverse field and the nearest neighbor interactions $\mathcal{I}_{i j}$ are independent random variables of mean zero) can be represented as the Hamiltonian of the independent quantum two-level systems (low energy droplets) of the form 


$$
\mathcal{H}=\frac{1}{2} \sum_{L}^{\sim} \sum_{D_{L}}\left(\epsilon_{D_{L}} S_{D_{L}}^{z}+\Gamma_{L} S_{D_{L}}^{x}\right)
$$

where $S_{D_{L}}^{z}$ and $S_{D_{L}}^{x}$ are the Pauli matrices representing the two states of the droplet; the sum is over all droplets $D_{L}$ at length scale $L$ and over all length scales $L$, and

$$
\sum_{L}^{\sim} \sim \int_{L_{0}}^{\infty} \frac{d L}{L}
$$

with a short-distance cutoff $L_{0}$. The droplet length scale $L$ is more or of the order of the correlation length. $\epsilon_{D_{L}}$ is the droplet energy which is independent random variable. The value $\Gamma_{L}$ regulates the strength of quantum fluctuations ( $\Gamma_{L} \rightarrow 0$ corresponds to the classical limit).

$$
\Gamma_{L}=\Gamma_{0} e^{-\sigma L^{d}}
$$

is the tunneling rate for a droplet of the linear size $L, \Gamma_{0}$ is the microscopic tunneling rate; $\sigma$ is defined from the equation $2 K=\sigma L^{d}$ where $2 K$ is the surface free energy of an interface between the two droplet states, so $\sigma$ is a reduced surface tension for this interface; $\sigma$ is approximately the same for all droplet. We will assume that $\Gamma_{L}$ is the same for all droplets of scale $L$. The Hamiltonian of a single droplet is the $(2 \times 2)$ matrix [6]

$$
\frac{1}{2}\left(\begin{array}{cc}
\epsilon_{D_{L}} & \Gamma_{L} \\
\Gamma_{L} & -\epsilon_{D_{L}}
\end{array}\right)
$$

with eigenvalues $E_{ \pm}= \pm \sqrt{\epsilon_{L}^{2}+\Gamma_{L}^{2}}$.

$E=2\left|E_{ \pm}\right|$is the energy difference between the two eigenvalues. Note the Hamiltonian (33) is similar to the Hamiltonian of two-level system in real glass [24]. The droplet excitations have a broad distribution of their free energies at scale $L$ for large $L$ in a scaling form $[4,6]$

$$
P_{L}\left(\epsilon_{L}\right) d \epsilon_{L}=\frac{d \epsilon_{L}}{\gamma(T) L^{\theta}} \mathcal{P}\left(\frac{\epsilon_{L}}{\gamma(T) L^{\theta}}\right), L \rightarrow \infty
$$

It is assumed that $P_{L}(x \rightarrow 0)>0, P_{L}(0)-P_{L}(x) \sim x^{\phi}$ at $x \rightarrow 0 . \gamma(T)$ is a generalized temperature dependent stiffness modulus which is of the order of characteristic exchange $\mathcal{I}={\overline{\left(\mathcal{I}_{i j}^{2}\right)}}^{1 / 2}$ at $T=0$ and vanishes for $T \geq T_{g}$.

There is a crossover length scale, $L^{*}(T)$, defined by condition $\Gamma_{L^{*}(T)}=k_{B} T$ or $L^{*}(T)=$ $\left[(1 / \sigma) \log \left(\Gamma_{0} / k_{B} T\right)\right]^{1 / d}$. For droplets with $L \ll L^{*}(T)$ and $\Gamma_{L} \gg k_{B} T$ the energy $\sqrt{\epsilon_{L}^{2}+\Gamma_{L}^{2}}$ is always more than $k_{B} T$ and thermal fluctuations are insignificant at temperature $T$. Droplets with $L \gg L^{*}(T)$ have $\Gamma_{L} \ll$ $k_{B} T$ and behave classically. The large droplets $\left(\epsilon_{L} \leq\right.$ $\left.k_{B} T, \Gamma_{L} \leq k_{B} T\right)$ are thermally active. At low $T$ only a small fraction of droplets is thermally active, but many low- $T$ static properties are dominated by these droplets at the crossover length $L^{*}(T)$.
The total magnet moment of a droplet wall scales as $q L^{\frac{d}{2}}$ where $q$ a random number with mean zero and

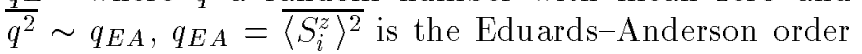
parameter [4]. The total magnetization $M$ of the sample will be

$$
M=\tilde{\sum_{L}} \frac{V}{L^{d}} \sum_{D_{L}}\left\langle S_{D_{L}}^{z}\right\rangle q L^{d / 2}
$$

and

$$
m=\frac{M}{V}=\tilde{\sum_{L}} \overline{\left\langle S_{D_{L}}^{z}\right\rangle q L^{-d / 2}}
$$

where $\overline{\left\langle S_{D_{L}}^{z}\right\rangle q L^{-d / 2}}$ means the average over the droplets energies $\epsilon_{L}$. The static $\chi_{3}$ susceptibilities are defined in terms of the expansion of magnetization into Taylor series approximately as

$$
M=\chi_{1} h-\chi_{3} h^{3}+\ldots
$$

For sufficiently small external field it is possible to be restricted by few terms of this expansion. The susceptibilities are obtained as derivatives with respect to $h$ at $h=0: \chi_{1}=\left.(\partial m / \partial h)\right|_{h=0}, \chi_{3}=\left.\left(\partial^{3} m / \partial h^{3}\right)\right|_{h=0}$.

M. J. Thill and D. A. Huse have calculated static linear and nonlinear susceptibilities for droplet system described by the Hamiltonian (30). The static linear susceptibility diverges at $T=0$ below the lower critical dimension $d_{l}$. The static nonlinear susceptibility diverges in all dimensions $d$. The static linear susceptibility appears to start away from the nonzero constant $T=0$ value decreasingly versus $T$ to the lowest order [6].

In this paper using the quantum droplet model of the short-range Ising spin glass in a transverse field and quantum-mechanical case $\beta \Gamma_{L} \gg 1$ (quantum regime) we calculate the third order nonlinear dynamic response at very low finite temperatures.

\section{QUANTUM DROPLET DYNAMIC NONLINEAR SUSCEPTIBILITY}

When we consider the droplets at finite temperatures they may have two characteristic rates, (a) the Rabi frequency (is of the order $\Gamma_{L}$ ) and (b) the rate of classical activation over energy barrier $B$ for the annihilation and creation of the droplet excitations [6]

$$
t \sim \tau_{0} \exp \left(\frac{B}{k_{B} T}\right)
$$

where $B \sim \Delta L^{\psi}, 0 \leq \psi \leq d-1, \psi$ is some exponent [4], $\Delta$ is a barrier energy at $t \ll T_{g}, \Delta \sim \mathcal{I}$; $\tau_{0}$ is a microscopic time. There is a complicated dynamical classicalto-quantum crossover depending on the temperature frequency of ac external field and length scale $L$. According 


\section{G. BUSIELLO, R. V. SABUROVA, V. G. SUSHKOVA}

to [6] the crossover dynamic length is determined from the condition $\Gamma_{L}^{-1}=T$, i. e.,

$$
L_{d y n}^{*}(T) \sim\left(\frac{\sigma}{\Delta} k_{B} T\right)^{1 /(\psi-d)}
$$

The system behaves presumably classically or quantum mechanically when the dominant length scale $L$ is above or below $L_{d y n}^{*}$ for the frequency $\omega$. The linear dynamic susceptibility for the model Hamiltonian (30) was calculated at zero temperature in [6]. This susceptibility is dominated by droplets at a length scale such that $\omega$ is of the order the characteristic frequency of the quantum droplet system $\Gamma_{L}$. We have calculated the linear dynamic susceptibility (real and imaginary parts) at finite temperatures considering both quantum and classical limit and found a glassy behaviour [16]. M. J. Thill and D. A. Huse [6] suppose that the nonlinear dynamic susceptibility that $\chi_{3}^{\prime}(3 \omega, \omega)$ diverges as a power of external field frequency $\omega$ at $T=0$ if $\phi<2$, in strong contrast to classical Ising spin glass at $T>0$, where it diverges as a power of $\log \omega[4]$ ( $\phi$ is a power of $\epsilon_{L}$ in the distribution of droplet energies $\epsilon_{L}$ at the length scale $L$ [6]).

Now we consider dynamic third-order susceptibility $\chi_{3}^{\prime}(\omega t, T)$ at finite very low temperatures (quantum regime) when $\beta \Gamma_{L} \gg 1$. We define nonlinear third order dynamic susceptibility $\chi_{3}^{\prime}(\omega, T)$ by expressions $(24)$ and (29)

$$
\begin{aligned}
& \chi_{3}^{\prime}(\omega, T)=\frac{1}{(i \hbar)^{3}} \frac{P_{3}}{3 !} \int_{0}^{\infty} d \tau_{1} \int_{0}^{\infty} d \tau_{2} \\
& \times \int_{0}^{\infty} d \tau_{3} \exp \left[i\left(3 \omega \tau_{1}+\omega \tau_{2}+\omega \tau_{3}\right)\right] \\
& \times\left\langle\left[\left[\left[M_{i}\left(\tau_{1}+\tau_{2}+\tau_{3}\right), M_{j}\left(\tau_{2}+\tau_{3}\right)\right], M_{k}\left(\tau_{3}\right)\right], M_{l}\right]\right\rangle
\end{aligned}
$$

We now consider the cubic dynamic nolinear susceptibility $\chi_{3}(\omega, T)$. The contribution of a single droplet to the real part of dynamic third-order susceptibility up to some factor $\sim q_{E A}^{2} L^{2 d}$ is proportional to

$$
\chi_{3 D_{L}}^{\prime} \sim q_{E A}^{2} \frac{\left(\sum_{k=0}^{5} A_{k}\left(\omega, \Gamma_{L}\right) \epsilon_{L}^{2 k}\right) \tanh \left(\frac{1}{2} \beta \sqrt{\epsilon_{L}^{2}+\Gamma_{L}^{2}}\right)}{\left(\epsilon_{L}^{2}+\Gamma_{L}^{2}\right)^{\frac{5}{2}}\left(\epsilon_{L}^{2}+\Gamma_{L}^{2}-9 \omega^{2}\right)\left(\epsilon_{L}^{2}+\Gamma_{L}^{2}-\omega^{2}\right)^{2}\left(\epsilon_{L}^{2}+\Gamma_{L}^{2}-\frac{\omega^{2}}{4}\right) \omega^{2}},
$$

where

$$
\begin{aligned}
A_{0} & =\omega^{2} \Gamma_{L}^{10}+4 \omega^{4} \Gamma_{L}^{8}-5 \omega^{6} \Gamma_{L}^{6} \\
A_{1} & =2 \Gamma_{L}^{10} \Gamma_{L}^{10}-12.5 \omega^{2} \Gamma_{L}^{8}-49.75 \omega^{4} \Gamma_{L}^{6} \\
& -23.5 \omega^{6} \Gamma_{L}^{4}-2.25 \omega^{8} \Gamma_{L}^{2} \\
A_{3} & =12 \Gamma_{L}^{6}-45.5 \omega^{2} \Gamma_{L}^{4}+41.75 \omega^{2} \Gamma_{L}^{4} \\
A_{4} & =8 \Gamma_{L}^{4}-15.5 \omega^{2} \Gamma_{L}^{2}
\end{aligned}
$$

and $A_{5}=2 \Gamma_{L}^{2}$.

We have to average $\chi_{3 D_{L}}$ over droplet energies $\epsilon_{L}$ using the distribution of droplet free energies (35) and changing variables from $\epsilon_{L}$ to $x=\beta \epsilon_{L}$. As follow froms (42) to average over droplet energies we have to distinguish cases: frequency $3 \omega$ greater or less than $\Gamma_{L}, \omega$ greater or less than $\Gamma_{L}$ and $\frac{\omega}{2}$ greater or less than $\Gamma_{L}$, then we have to average the obtained expression over all length scales $L$; see Eq. (32).

In our calculation we take into accout the first two terms in the expansion of

$$
\tanh \left(\frac{1}{2} \sqrt{x^{2}+\beta^{2} \Gamma_{L}^{2}}\right) \simeq 1-2 e^{-\sqrt{x^{2}+\beta^{2} \Gamma_{L}^{2}}}
$$

and apply the approximation

$$
\sqrt{x^{2}+\beta^{2} \Gamma_{L}^{2}} \simeq \beta \Gamma_{L}+\frac{x^{2}}{2 \beta \Gamma_{L}}
$$

because we have $\beta \Gamma_{L} \gg 1$.

After averaging over droplet energies for cases $\Gamma_{L}>$ $3 \omega, \Gamma_{L} \sim 3 \omega, \Gamma_{L}<3 \omega$ we have received that the real part of the nonlinear susceptibility is dominated by droplets of length scale

$$
L_{\mathrm{dom}(3 \omega)} \sim\left(\frac{1}{\sigma}\left|\log \left(\frac{3 \omega}{\Gamma_{0}}\right)\right|\right)^{1 / d}
$$

which is determined by condition $\Gamma_{L} \sim 3 \omega$. Then for $\Gamma_{L}>3 \omega$ the result of two averages is given by the following expression 


$$
\begin{aligned}
& \chi_{3}^{\prime} \sim \frac{q_{E A}^{2}}{\gamma^{1+\phi}}\left\{\pi \sec \left[\frac{\pi \phi}{2}\right] \sum_{k=-2,0,2} A_{k} \omega^{k-2} \Gamma_{0}^{\phi-k} \frac{[\sigma(\phi-k)]^{-\alpha}}{d} \mathrm{G}\left[\alpha,\left|\log \left(\frac{3 \omega}{\Gamma_{0}}\right)\right|(\phi-k)\right]\right. \\
& \left.+\frac{1}{\alpha d}\left(\frac{1}{\sigma}\left|\log \left(\frac{3 \omega}{\Gamma_{0}}\right)\right|\right)^{\alpha} e^{-3 \beta \omega} \sum_{k=0,1} B_{k} \omega^{(\phi-5-2 k) / 2} \beta^{-(\phi+1+2 k) / 2}\right\},
\end{aligned}
$$

where $\mathrm{G}[\alpha, z]$ is incomplete gamma-function. The coefficients in this expression depend on $\phi$ only and are given in Appendix A. Nonlinear susceptibility given by expression (44) does not diverge if $2<\phi<3$ and $-1+\frac{d}{\theta}<\phi$. For case $\Gamma_{L}<3 \omega$ we obtained a similar expression (Appendix A).

After averaging over droplet energies for cases $\Gamma_{L}>\omega, \Gamma_{L} \sim \omega, \Gamma_{L}<\omega$ we have received that the real part of the nonlinear susceptibility is dominated by droplets of length scale

$$
L_{\mathrm{dom}(\omega)} \sim\left(\frac{1}{\sigma}\left|\log \left(\frac{\omega}{\Gamma_{0}}\right)\right|\right)^{1 / d}
$$

which is determined by condition $\Gamma_{L} \sim \omega$. For example, for the case of $\Gamma_{L}<\omega$ we received as a result of two averages

$$
\begin{aligned}
\chi_{3}^{\prime} & \sim \frac{q_{E A}^{2}}{\gamma^{1+\phi}}\left\{\frac{1}{\alpha d}\left(\frac{1}{\sigma}\left|\log \left(\frac{\omega}{\Gamma_{0}}\right)\right|\right)^{\alpha} N_{0} \omega^{\phi-2}+\sum_{k=1}^{8} N_{k} \omega^{\phi-2-k} \Gamma_{0}^{k} \frac{(\sigma k)^{-\alpha}}{d} \mathrm{G}\left[\alpha,\left|\log \left(\frac{\omega}{\Gamma_{0}}\right)\right| k\right]\right. \\
& +\pi \sec \left[\frac{\pi \phi}{2}\right] \sum_{k=0,2} R_{k} \omega^{k-2} \Gamma_{0}^{\phi-k} \frac{(\sigma(\phi-k))^{-\alpha}}{d} \mathrm{G}\left[\alpha,\left|\log \left(\frac{\omega}{\Gamma_{0}}\right)\right|(\phi-k)\right] \\
& \left.+\frac{1}{\alpha d}\left(\frac{1}{\sigma}\left|\log \left(\frac{\omega}{\Gamma_{0}}\right)\right|\right)^{\alpha} e^{-\beta \omega} \sum_{k=0}^{2} P_{k} \omega^{(\phi-5-2 k) / 2} \beta^{-(\phi+1+2 k) / 2}\right\} .
\end{aligned}
$$

The coefficients in this expression depend on $\phi$ only and are given in Appendix B. Nonlinear susceptibility given by expression (46) does not diverge if $2<\phi<7$ and $(-1+d / \theta)<\phi$. (The cases of $\Gamma_{L}>\omega$ and $\Gamma_{L} \sim \omega$ are given in Appendix B).

After integration over droplet energies for cases $\Gamma_{L}>\frac{\omega}{2}, \Gamma_{L} \sim \frac{\omega}{2}, \Gamma_{L}<\frac{\omega}{2}$ we have received that the real part of the nonlinear susceptibility is dominated by droplets of length scale

$$
L_{\mathrm{dom}\left(\frac{\omega}{2}\right)} \sim\left(\frac{1}{\sigma}\left|\log \left(\frac{\omega}{2 \Gamma_{0}}\right)\right|\right)^{1 / d}
$$

which is determined by condition $\Gamma_{L} \sim \frac{\omega}{2}$. Then for case $\Gamma_{L}>\frac{\omega}{2}$ the result of two averages is given by the following expression

$$
\begin{aligned}
& \chi_{3}^{\prime} \sim \frac{q_{E A}^{2}}{\gamma^{1+\phi}}\left\{\frac{1}{\alpha d}\left(\frac{1}{\sigma}\left|\log \left(\frac{\omega}{2 \Gamma_{0}}\right)\right|\right)^{\alpha} T_{0} \omega^{\phi-2}+\sum_{k=1}^{8} T_{k} \omega^{\phi-2-k} \Gamma_{0}^{k} \frac{(\sigma(\phi-k))^{-\alpha}}{d} \mathrm{G}\left[\alpha, \log \left(\frac{\omega}{2 \Gamma_{0}}\right) \mid(\phi-k)\right]\right. \\
& +\pi \sec \left[\frac{\pi \phi}{2}\right] \sum_{k=0,2} S_{k} \omega^{k-2} \Gamma_{0}^{\phi-k} \frac{(\sigma(\phi-k))^{-\alpha}}{d} \mathrm{G}\left[\alpha,\left|\log \left(\frac{\omega}{2 \Gamma_{0}}\right)\right|(\phi-k)\right] \\
& \left.+\frac{1}{\alpha d}\left(\frac{1}{\sigma}\left|\log \left(\frac{\omega}{2 \Gamma_{0}}\right)\right|\right)^{\alpha}\left(e^{-\beta \omega} \sum_{k=0}^{2} U_{k} \omega^{(\phi-5-2 k) / 2} \beta^{-(\phi+1+2 k) / 2}+e^{-(3 \beta \omega) / 4} U_{3} \omega^{\phi-3} \beta^{-1}\right)\right\} .
\end{aligned}
$$

The coefficients in this expression depend on $\phi$ only and are given in Appendix C. Nonlinear susceptibility given by expression (48) does not diverge if $2<\phi<7$ and $\left(-1+d / \theta<\phi\right.$. (Cases $\Gamma_{L} \sim(\omega / 2), \Gamma_{L}<(\omega / 2)$ are given in Appendix C). 
Expressions for susceptibility consist of two parts: one part does not depend on temperature, the other one depends on temperature. Nonlinear susceptibility has strong dependence on distribution function $P_{L}\left(\epsilon_{L}\right)$, i. e. on $\phi$, on droplet microscopic tunneling rate $\Gamma_{0}$ and other parameters. One can see that the real part $\chi_{3}^{\prime}(\omega, T)$ varies approximately logarithmically with frequency. This signalizes broad distribution of relaxation times of the system.

Let us take for numerical calculation the following numbers: $\theta=1, \Gamma_{0}=10^{10} \mathrm{~s}^{-1}, d=3, \phi=2.5, \gamma=$ $10^{-15} \mathrm{erg}, \sigma=10^{-15}, q=0.5$. The frequency dependence of $\chi_{3}^{\prime}(\omega, T)$ is shown in Fig. 1. We give $\log _{10} f$ dependence at $\log _{10} f$ from 0 to 11 at several fixed temperatures: $T_{1}=0.001, T_{2}=0.005, T_{3}=0.01, T_{4}=0.05$.

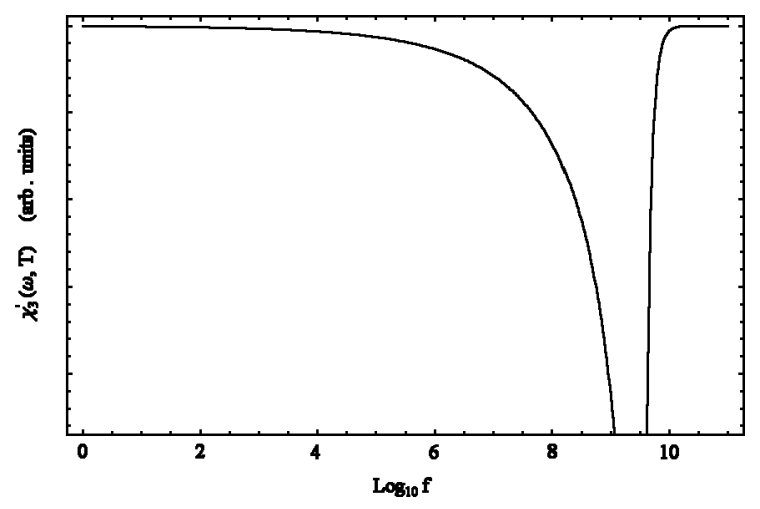

Fig. 1. The frequency dependence of real of the nonlinear dynamical susceptibility $\chi_{3}^{\prime}(\omega, T)$ versus $\log _{10} f$ at fixed temperature

The frequency interval covers some decades of frequencies. Our numerical calculations show the crossover between low $\omega$ and high- $\omega$ behaviours. In low- $\omega$ region the nonlinear response is found nonsingular and slowly decreasing. When the frequency increases the curve falls down more quickly, the nonlinear response diverges at $\omega \sim \Gamma_{0} / 3$, then the curve rises to some value. In low- $\omega$ region we have a qualitative agreement with experimental data for disordered dipolar magnet $\mathrm{LiHo}_{x} \mathrm{Y}_{1-x} \mathrm{~F}_{4}$. At different low fixed temperatures the behaviour of $\chi_{3}^{\prime}(\omega, T)$ is the same and the values of $\chi_{3}^{\prime}(\omega, T)$ are approximately the same. Therefore we give only one curve for all fixed temperatures.

In Fig. 2 we give the temperature dependence of $\chi_{3}^{\prime}(\omega, T)$ at the temperatures from 0 to $10^{-2} \mathrm{~K}$ at several fixed frequencies: $f_{1}=10^{7} \mathrm{~Hz}, f_{2}=2.510^{7} \mathrm{~Hz}, f_{3}=$ $510^{7} \mathrm{~Hz}, f_{4}=7.510^{7} \mathrm{~Hz}, f_{5}=10^{8} \mathrm{~Hz}$ of ac field $(f=$ $\omega / 2 \pi)$. The behaviour of $\chi_{3}^{\prime}(\omega, T)$ indicates the following glassy-like features. The curves of the temperature dependence of $\chi_{3}^{\prime}(\omega, T)$ have maxima depending on fixed frequency. The temperature of $\chi_{3}^{\prime}$-maximum $T_{f}(\omega)$ depends on frequency. The nonlinear susceptibility magnitudes at different fixed frequencies are remarkably distinguishable. The temperatures of maximum values are different. When the frequency increases the temperature of $\chi_{3}^{\prime}$-maximum shifts towards high temperatures. The similar curve of temperature variation of $\chi_{3}^{\prime}$ was observed in spin glasses at higher temperatures $[2,13]$. If we consider only the $T$-dependent part of $\chi_{3}^{\prime}$, we will see that the $\chi_{3}^{\prime}$-maxima are sharp (Fig. 3).

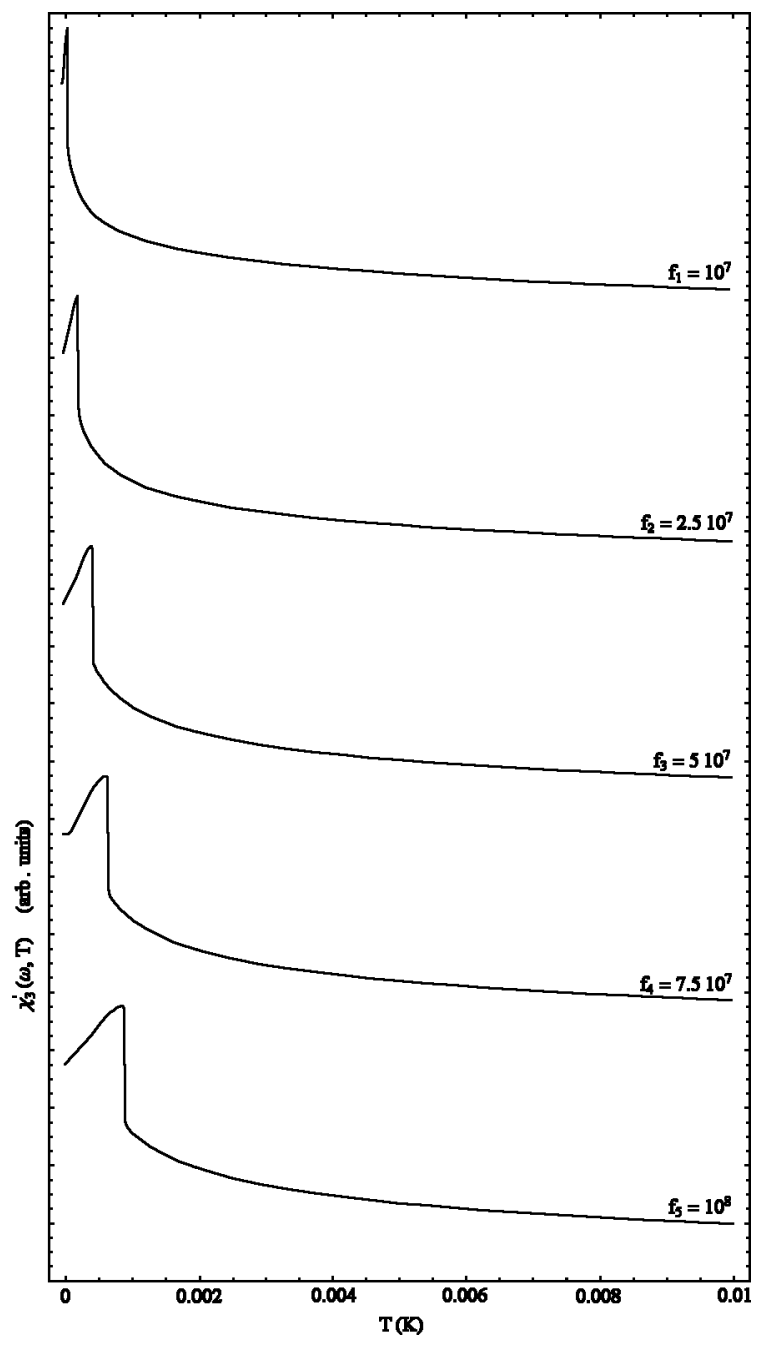

Fig. 2. The temperature dependence of the real part of the $\chi_{3}^{\prime}(\omega, T)$ at various frequencies $f$.

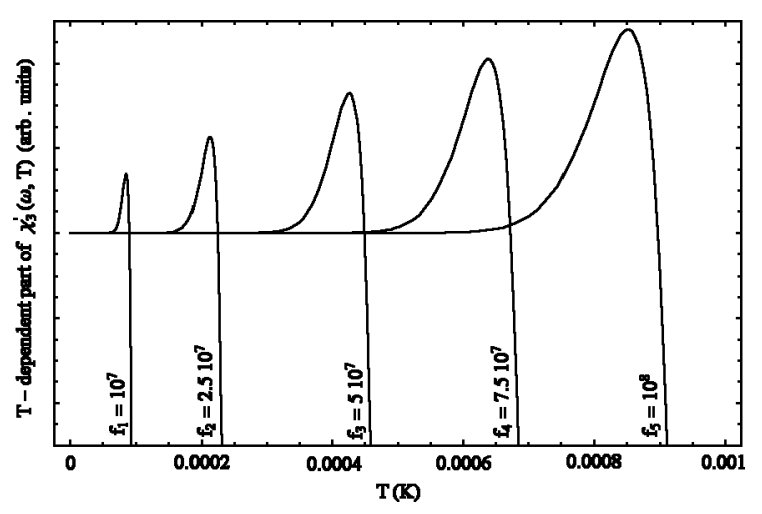

Fig. 3. The temperature dependence of the $T$-dependent part of the $\chi_{3}^{\prime}(\omega, T)$ at various frequencies $f$. 


\section{DISCUSSION AND CONCLUSION}

The cubic dynamic susceptibility $\chi_{3}^{\prime}(3 \omega)$ is analitically and numerically calculated in quantum spin glass in terms of quantum droplet model on the basis of general dynamic nonlinear quantum-mechanical response theory. We have carefully analyzed the susceptibility temperature-frequency behaviour to study the properties of the low temperature magnetic state and to determine whether or not a conventional spin glass state exists below $T_{f}$. Comparing with the case of a true spin glass transition we see that our data indicate that the magnetic state below $T_{f}$ does not correspond to a conventional spin glass state below $T_{f}$. We find a glassy type slow dynamics. Our calculations show that the $a c$ nonlinear susceptibilities scale for $\Gamma_{L}>3 \omega$ with such a frequency as

$$
\begin{aligned}
& \chi_{3}^{\prime}(3 \omega) \sim \sum_{k=0,1} A(\mathbf{k}) \omega^{(\phi-5-2 k) / 2} e^{-3 \beta \omega} \\
& \times\left|\log \left(3 \omega \Gamma_{0}^{-1}\right)\right|^{[d-(1+\phi) \theta] / \phi} \\
& +\sum_{n=-2,0,2} B(n) \omega^{n-2} \mathrm{G}\left[\alpha,\left|\log \left(3 \omega \Gamma_{0}^{-1}\right)\right|(\phi-n)\right],
\end{aligned}
$$

where $A(\mathbf{k})$ and $B(n)$ and some expressions which do not contain $\omega$. The nonlinear susceptibility diverges for $\omega \rightarrow 0$ if $d>(1+\phi) \theta$ and $\phi<7$, in contrast to classical Ising spin glass at $T>0$ (where it diverges as a power of $\log \omega)$. Similar frequency dependence was observed by W. Wu et al. [14]. So, the droplet dynamics at very low temperatures is extremely slow.

Our calculations at $T=0$ coincide with $T=0$ result of M. J. Thill and D. A. Huse [6]. For finite temeratures we find some features which have been recently observed [13]. We suppose that at some very low temperature (temperature of the maximum of $\chi_{3}^{\prime}(\omega, T)$ ) there is a phase transition. If $\theta>0$ and $d=3$, we suppose a true phase transition at very low temperature $T_{f}$. For our numerical data we find $T_{f} \sim 10^{-4} \div 8.510^{-4} \mathrm{~K}$ for $f=10^{7} \div 10^{8} \mathrm{~Hz}$, respectively (Fig. 3)

Besides frequency and temperature dependence the shape of $\chi_{3}^{\prime}(3 \omega)$ depends crucially on the probability distribution of droplet free energies, on the tunneling rate for a droplet of linear size $L$, on the material parameters. In consequence of this dependence there is divergence (or convergence) of $\chi_{3}^{\prime}(3 \omega)$. We need to take into account (in a future paper) the dipole-dipole interaction between droplets and also droplet lattice interaction.

Applying our results to the reported experimental data on the nonlinear dynamic susceptibility of $\mathrm{LiHo}_{x} \mathrm{Y}_{1-x} \mathrm{~F}_{4}$ we demonstrate that a fairly good agreement may be achieved.

This work is partially supported by the RBRF under Grant 01-02-16368.

\section{APPENDIX A}

The coefficients in expression (44) are the following:

$$
\begin{aligned}
& \alpha=\frac{d-\theta(1+\phi)}{d} ; \\
& A_{-2}=-\frac{1}{4}-\frac{\phi}{4}, A_{0}=-\frac{6263}{1600}-\frac{23 \phi}{100}+\frac{6441}{6400} 2^{\frac{\phi+1}{2}}, A_{2}=-\frac{135}{10}+122^{\frac{\phi+1}{2}}, \\
& B_{0}=\mathrm{G}\left[\frac{\phi-1}{2}\right] \frac{2^{\frac{\phi-7}{2}} 3^{\frac{\phi-1}{2}}\left(1435 \phi^{2}+843 \phi-1988\right)}{175}, B_{1}=\mathrm{G}\left[\frac{\phi-1}{2}\right] \frac{2^{\frac{\phi-7}{2}} 3^{\frac{\phi-3}{2}}\left(791-791 \phi^{2}\right)}{5} . \\
& \chi_{3}^{\prime}(\omega, T) \sim \frac{q_{E A}^{2}}{\gamma^{1+\phi}} \frac{\left(\frac{1}{\sigma}\left|\log \left(\frac{3 \omega}{\Gamma_{0}}\right)\right|\right)^{\alpha}}{\alpha d}\left\{C_{0} \pi \sec \left[\frac{\pi \phi}{2}\right] \omega^{\phi-2}+e^{-3 \beta \omega} \sum_{k=1}^{4} C_{k} \omega^{\frac{\phi+1-2 k}{2}} \beta^{\frac{-\phi+5-2 k}{2}}\right\}
\end{aligned}
$$

for $\Gamma_{L} \sim 3 \omega$ where

$$
\begin{aligned}
& C_{0}=\frac{24335^{\frac{\phi-1}{2}} 2^{7-\phi}}{185}-\frac{689312^{3 \phi-3}}{925}+\frac{3^{\phi}}{2}-\frac{3912^{\frac{\phi+5}{2}} 3^{\phi}}{925}-\frac{272^{\frac{3 \phi-5}{2}} \phi}{5}, C_{1}=-\mathrm{G}\left[\frac{\phi-1}{2}\right] \frac{2^{\frac{\phi-3}{2}} 3^{\frac{\phi+3}{2}}}{5}, \\
& C_{2}=\mathrm{G}\left[\frac{\phi-1}{2}\right] \frac{2^{\frac{\phi-3}{2}} 3^{\frac{\phi+1}{2}}}{5}-\mathrm{G}\left[\frac{\phi+1}{2}\right] \frac{2^{\frac{\phi+1}{2}} 3^{\frac{\phi-5}{2}}(458+945 \phi)}{35}
\end{aligned}
$$




$$
\begin{aligned}
& C_{3}=-\mathrm{G}\left[\frac{\phi+1}{2}\right] \frac{118972^{\frac{\phi-7}{2}} 3^{\frac{\phi-5}{2}}}{175}+\mathrm{G}\left[\frac{\phi+3}{2}\right] \frac{102062^{\frac{\phi-7}{2}} 3^{\frac{\phi-5}{2}}}{35}, \\
& C_{4}=-\mathrm{G}\left[\frac{\phi+3}{2}\right] \frac{3591062^{\frac{\phi-7}{2}} 3^{\frac{\phi-5}{2}}}{175} .
\end{aligned}
$$

This expression does not diverge if $2<\phi<3$ and $-1+\frac{d}{\theta}<\phi$. This expression has singularity at $\omega \sim \frac{\Gamma_{0}}{3}$. When the frequency increases the values of $\chi_{3}^{\prime}$ are growing to infinity while $\omega \rightarrow \frac{\Gamma_{0}}{3} \cdot \chi_{3}^{\prime}$ maintains this property when $\omega$ is more than $\frac{\Gamma_{0}}{3}$. The temperature dependence of $\chi_{3}^{\prime}$ in this case has no extremes, we observe monotonous decrease of values of $\chi_{3}^{\prime}$ with temperature.

\section{APPENDIX B}

The coefficients in expression (46) are the following:

$$
\begin{aligned}
& N_{0}=\frac{576 \phi^{2}+2223 \phi+3015}{405(\phi+1)(\phi+3)}, \quad N_{1}=\frac{4941 \phi^{2}+7586 \phi+4589}{405(\phi+1)(\phi+3)}, \quad N_{2}=\frac{-3293 \phi^{2}-1994 \phi+2595}{405(\phi+1)(\phi+3)}, \\
& N_{3}=-\frac{2595 \phi^{2}+4156 \phi+1553}{405(\phi+1)(\phi+3)}, \quad N_{4}=\frac{1641 \phi^{2}+1294 \phi-347}{405(\phi+1)(\phi+3)}, \quad N_{5}=\frac{499 \phi^{2}+819 \phi+296}{405(\phi+1)(\phi+3)}, \\
& N_{6}=\frac{-312 \phi^{2}-266 \phi+30}{405(\phi+1)(\phi+3)}, \quad N_{7}=-\frac{6 \phi+4}{81(\phi+3)}, \quad N_{8}=\frac{4 \phi}{81(\phi+3)}, \\
& R_{0}=\frac{4\left(2^{\frac{\phi-1}{2}}-1\right)}{25}, \quad R_{2}=\frac{\phi-11}{50}, \quad P_{0}=-\mathrm{G}\left[\frac{\phi+1}{2}\right] \frac{2^{\frac{\phi-5}{2}}(24 \phi+85)}{3}, \quad P_{1}=\mathrm{G}\left[\frac{\phi+3}{2}\right] \frac{77872^{\frac{\phi-9}{2}}}{75}, \\
& P_{2}=\left(\mathrm{G}\left[\frac{\phi+3}{2}\right]+\mathrm{G}\left[\frac{\phi+5}{2}\right]\right) \frac{2^{\frac{\phi-9}{2}} 374410(\phi+3)}{225(\phi+5)} . \\
& \chi_{3}^{\prime} \sim \frac{q_{E A}^{2}}{\gamma^{1+\phi}}\left\{\frac{\left(\frac{1}{\sigma}\left|\log \left(\frac{\omega}{\Gamma_{0}}\right)\right|\right)^{\alpha}}{\alpha d} J_{0} \omega^{\phi-2}+\sum_{k=1}^{3} J_{k} \omega^{\phi-2-k} \Gamma_{0}^{k} \frac{(\sigma k)^{-\alpha}}{d} \mathrm{G}\left[\alpha,\left|\log \left(\frac{\omega}{\Gamma_{0}}\right)\right| k\right]\right. \\
& +\pi \sec \left[\frac{\pi \phi}{2}\right] \sum_{k=0,2} K_{k} \omega^{k-2} \Gamma_{0}^{\phi-k} \frac{(\sigma(\phi-k))^{-\alpha}}{d}\left[\mathrm{G}\left[\alpha,\left|\log \left(\frac{\omega}{\Gamma_{0}}\right)\right|(\phi-k)\right]\right. \\
& \left.+\frac{\left(\frac{1}{\sigma}\left|\log \left(\frac{\omega}{\Gamma_{0}}\right)\right|\right)^{\alpha}}{\alpha d}\left(e^{-\beta \omega} \sum_{k=0}^{3} L_{k} \omega^{\frac{\phi-3-2 k}{2}} \beta^{\frac{-\phi+1-2 k}{2}}+e^{-3 \beta \omega} L_{4} \omega^{\phi-3} \beta^{-1}\right)\right\}
\end{aligned}
$$

for $\Gamma_{L}>\omega$ where

$$
\begin{aligned}
& J_{0}=-\frac{3^{\phi}\left(4 \phi^{2}-36 \phi-34\right)}{5(\phi+1)(\phi+3)}, \quad J_{1}=\frac{3^{\phi-1}\left(26 \phi^{2}+58 \phi+30\right)}{5(\phi+1)(\phi+3)}, \quad J_{2}=-\frac{3^{\phi-2}(6 \phi+2)}{\phi+3}, \quad J_{3}=\frac{3^{\phi-3} 2 \phi}{\phi+3}, \\
& K_{0}=\phi^{2}-\frac{157 \phi}{160}-\frac{103}{32}+\frac{2^{\frac{\phi-1}{2}} 21}{5}, \quad K_{2}=-\frac{3 \phi^{2}}{4}+\frac{2 \phi}{5}-\frac{37}{20}+\frac{2^{\frac{\phi+1}{2}} 8}{5}, \\
& L_{0}=\mathrm{G}\left[\frac{\phi+1}{2}\right] 2^{\frac{\phi-7}{2}}\left(9-\phi^{2}\right), \quad L_{1}=\mathrm{G}\left[\frac{\phi+1}{2}\right] \frac{2^{\frac{\phi-5}{2}}\left(-3 \phi^{3}-9 \phi^{2}+3 \phi-4\right)}{3}, \\
& L_{2}=\mathrm{G}\left[\frac{\phi+3}{2}\right] \frac{2^{\frac{\phi-9}{2}} 66601}{225}, \quad L_{3}=\mathrm{G}\left[\frac{\phi+5}{2}\right] \frac{2^{\frac{\phi-7}{2}}}{45}, \quad L_{4}=-\frac{2^{\phi+\frac{7}{2}} 7}{25} .
\end{aligned}
$$


This expression does not diverge if $2<\phi<5$ and $-1+\frac{d}{\theta}<\phi$.

$$
\chi_{3}^{\prime} \sim \frac{q_{E A}^{2}}{\gamma^{1+\phi}} \frac{\left(\frac{1}{\sigma}\left|\log \left(\frac{\omega}{\Gamma_{0}}\right)\right|\right)^{\alpha}}{\alpha d}\left\{\pi \sec \left[\frac{\pi \phi}{2}\right] M_{0} \omega^{\phi-2}+e^{-\beta \omega} \sum_{k=1}^{5} M_{k} \omega^{\frac{\phi+3-2 k}{2}} \beta \frac{-\phi+7-2 k}{2}\right\}
$$

for $\Gamma_{L} \sim \omega$ where

$$
\begin{aligned}
& M_{0}=-\frac{2^{\frac{\phi-1}{2}} 33}{50}-\frac{2^{8-\phi} 3^{\frac{\phi-1}{2}}}{50}+\frac{\phi+3}{10}, \quad M_{1}=\mathrm{G}\left[\frac{\phi+1}{2}\right] 2^{\frac{\phi+1}{2}}, \quad M_{2}=\mathrm{G}\left[\frac{\phi-1}{2}\right] 2^{\frac{\phi-1}{2}}(2-\phi), \\
& M_{3}=\mathrm{G}\left[\frac{\phi+1}{2}\right] 2^{\frac{\phi+3}{2}}, \quad M_{4}=-\mathrm{G}\left[\frac{\phi+1}{2}\right] \frac{2^{\frac{\phi-1}{2}}(30 \phi+121)}{15}, \quad M_{5}=\mathrm{G}\left[\frac{\phi+3}{2}\right] \frac{2^{\frac{\phi+1}{2}} 101}{45} .
\end{aligned}
$$

This expression does not diverge if $2<\phi<5$ and $-1+\frac{d}{\theta}<\phi$. Nonlinear susceptibility as a function of $\omega$ for the case of $\Gamma_{L}>\omega$ has singularity at $\omega \sim \Gamma_{0}$. Before this point we find $\chi_{3}^{\prime}$-minima depending on fixed temperature. When $\omega$ becomes larger than $\Gamma_{0}$ the values of $\chi_{3}^{\prime}$ grow to infinity (Fig. 4).

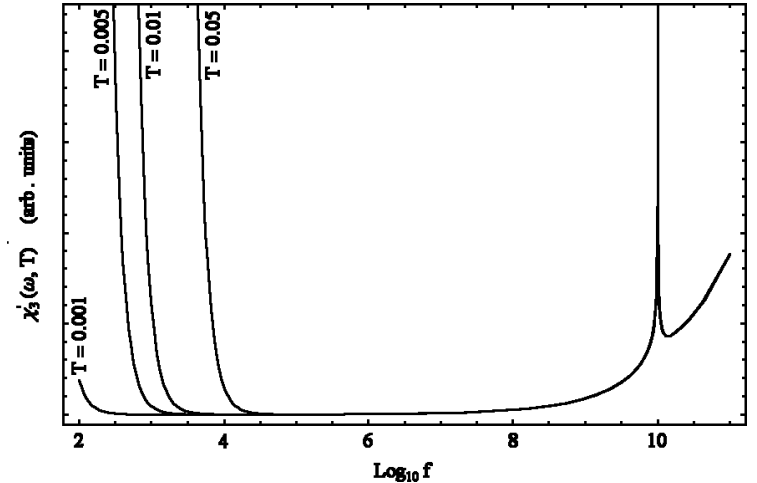

Fig. 4. The frequency dependence of the real part of the nonlinear dynamical susceptibility $\chi_{3}^{\prime}(\omega, T)$ versus $\log _{10} f$ at several fixed temperatures for case $\Gamma_{L}>\omega$

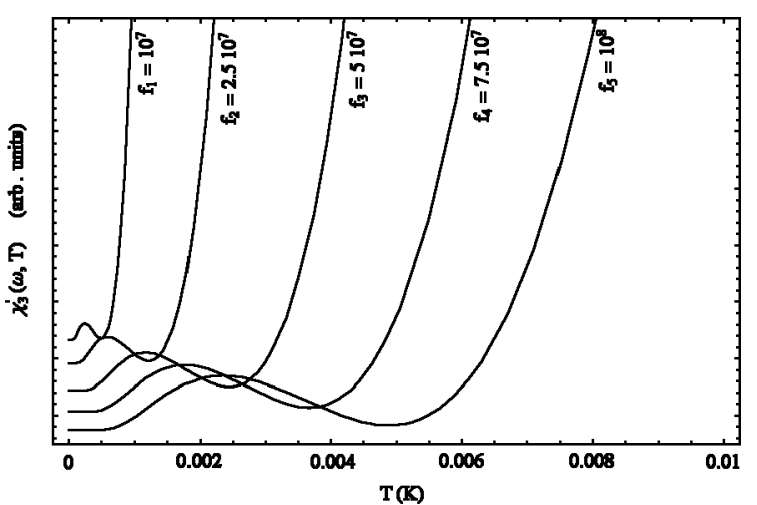

Fig. 5. The temperature dependence of the real part of the nonlinear dynamical susceptibility $\chi_{3}^{\prime}(\omega, T)$ at several fixed frequencies $f$.

In the temperature behaviour of $\chi_{3}^{\prime}$ we also observe the minima. The temperatures of the minima and their values depend on fixed frequencies.

The case of $\Gamma_{L} \sim \omega$ has its own particular features, for example in temperature dependence (Fig. 5).

\section{APPENDIX C}

The coefficients in expression (48) are the following:

$$
\begin{aligned}
& T_{0}=\frac{-3996 \phi^{2}-9548 \phi-6155}{405(\phi+1)(\phi+3)}, \quad T_{1}=\frac{4941 \phi^{2}+7586 \phi+4589}{405(\phi+1)(\phi+3)}, \quad T_{2}=\frac{-3293 \phi^{2}-1994 \phi+2595}{405(\phi+1)(\phi+3)} \\
& T_{3}=-\frac{2595 \phi^{2}+4156 \phi+1553}{405(\phi+1)(\phi+3)}, \quad T_{4}=\frac{1641 \phi^{2}+1294 \phi-347}{405(\phi+1)(\phi+3)}, \quad T_{5}=\frac{499 \phi^{2}+819 \phi+296}{405(\phi+1)(\phi+3)}, \\
& T_{6}=\frac{-312 \phi^{2}-266 \phi+30}{405(\phi+1)(\phi+3)}, \quad T_{7}=-\frac{6 \phi+4}{81(\phi+3)}, \quad T_{8}=\frac{4 \phi}{81(\phi+3)}
\end{aligned}
$$




$$
\begin{aligned}
& S_{0}=\frac{2^{\frac{\phi+1}{2}}-2}{5}, S_{2}=\frac{1-\phi}{20}, \quad U_{0}=-\mathrm{G}\left[\frac{\phi+1}{2}\right] \frac{1050 \phi+1814}{525}, \quad U_{1}=\mathrm{G}\left[\frac{\phi+3}{2}\right] \frac{31105904}{2039625} \\
& U_{2}=\left(\mathrm{G}\left[\frac{\phi+3}{2}\right]+\mathrm{G}\left[\frac{\phi+5}{2}\right]\right) \frac{777424(\phi+3)}{244755(\phi+5)}, \quad U_{3}=-\frac{2^{3-\phi} 806947}{6118875} \\
& \chi_{3}^{\prime} \sim \frac{q_{E A}^{2}}{\gamma^{1+\phi}} \frac{\left(\frac{1}{\sigma}\left|\log \left(\frac{\omega}{2 \Gamma_{0}}\right)\right|\right)^{\alpha}}{\alpha d}\left\{\pi \sec \left[\frac{\pi \phi}{2}\right] V_{0} \omega^{\phi-2}+e^{-\frac{\beta \omega}{2}} \sum_{k=1}^{5} V_{k} \omega^{\frac{\phi+3-2 k}{2}} \beta \frac{-\phi+7-2 k}{2}\right\}
\end{aligned}
$$

for $\Gamma_{L} \sim \frac{\omega}{2}$ where

$$
\begin{aligned}
& V_{0}=\frac{2^{1-\phi}(\phi-1)-2^{\frac{1-\phi}{2}}}{5}, \quad V_{1}=-\mathrm{G}\left[\frac{\phi+1}{2}\right] \frac{4}{5}, \quad V_{2}=\mathrm{G}\left[\frac{\phi+1}{2}\right] \frac{8}{5}, \\
& V_{3}=-\mathrm{G}\left[\frac{\phi+1}{2}\right] \frac{4}{5}, \quad V_{4}=\mathrm{G}\left[\frac{\phi+1}{2}\right] \frac{-3330 \phi-1646}{1665}, \quad V_{5}=\mathrm{G}\left[\frac{\phi+3}{2}\right] \frac{113788}{8325} .
\end{aligned}
$$

This expression does not diverge if $2<\phi<7$ and $-1+\frac{d}{\theta}<\phi$.

$$
\begin{aligned}
\chi_{3}^{\prime} & \sim \frac{q_{E A}^{2}}{\gamma^{1+\phi}}\left\{\frac{\left(\frac{1}{\sigma}\left|\log \left(\frac{\omega}{2 \Gamma_{0}}\right)\right|\right)^{\alpha}}{\alpha d} W_{0} \omega^{\phi-2}+\sum_{k=1}^{1} 3 W_{k} \omega^{\phi-2-k} \Gamma_{0}^{k} \frac{(\sigma k)^{-\alpha}}{d} \mathrm{G}\left[\alpha,\left|\log \left(\frac{\omega}{2 \Gamma_{0}}\right)\right| k\right]\right. \\
& \left.+\frac{\left(\frac{1}{\sigma}\left|\log \left(\frac{\omega}{2 \Gamma_{0}}\right)\right|\right)^{\alpha}}{\alpha d} e^{-\frac{\beta \omega}{2}} \sum_{k=0}^{2} X_{k} \omega^{\frac{\phi-5-2 k}{2}} \beta^{\frac{-\phi-1-2 k}{2}}\right\}
\end{aligned}
$$

for $\Gamma_{L}<\frac{\omega}{2}$ where

$$
\begin{aligned}
& W_{0}=-\frac{2^{-\phi-3}\left(306 \phi^{2}-2772 \phi-3044\right)}{81(\phi+1)(\phi+3)}, \quad W_{1}=\frac{2^{-\phi-3}\left(4644 \phi^{2}+11797 \phi-8001\right)}{81(\phi+1)(\phi+3)}, \\
& W_{2}=\frac{2^{-\phi-3}\left(-13014 \phi^{2}-23188 \phi-7583\right)}{81(\phi+1)(\phi+3)}, \quad W_{3}=\frac{2^{-\phi-3}\left(9420 \phi^{2}+12277 \phi-1015\right)}{81(\phi+1)(\phi+3)}, \\
& W_{4}=\frac{2^{-\phi-3}\left(5806 \phi^{2}+15864 \phi+10058\right)}{81(\phi+1)(\phi+3)}, \quad W_{5}=\frac{2^{-\phi-3}\left(-27668 \phi^{2}-50148 \phi-32432\right)}{81(\phi+1)(\phi+3)}, \\
& W_{6}=\frac{2^{-\phi-3}\left(60064 \phi^{2}+82368 \phi+22304\right)}{81(\phi+1)(\phi+3)}, \quad W_{7}=\frac{2^{-\phi-3}\left(-35008 \phi^{2}-35804 \phi+7940\right)}{81(\phi+1)(\phi+3)} \\
& W_{8}=\frac{2^{-\phi-3}\left(-17608 \phi^{2}-21024 \phi-3416\right)}{81(\phi+1)(\phi+3)}, \quad W_{9}=\frac{2^{-\phi-3}\left(10288 \phi^{2}+6448 \phi-448\right)}{81(\phi+1)(\phi+3)} \\
& W_{10}=\frac{2^{-\phi-3}\left(768 \phi^{2}+1280 \phi+512\right)}{81(\phi+1)(\phi+3)}, \quad W_{11}=\frac{2^{-\phi-3}\left(-768 \phi^{2}-1220 \phi-192\right)}{81(\phi+1)(\phi+3)}, \\
& W_{12}=\frac{2^{-\phi-3}\left(384 \phi^{2}+512 \phi+128\right)}{81(\phi+1)(\phi+3)}, \quad W_{13}=\frac{2^{-\phi-3}\left(-256 \phi^{2}-256 \phi\right)}{81(\phi+1)(\phi+3)}, \\
& X_{0}=\mathrm{G}\left[\frac{\phi+1}{2}\right] \frac{-1050 \phi-134}{525}, \quad X_{1}=\mathrm{G}\left[\frac{\phi+3}{2}\right] \frac{70266704}{2039625}, \quad X_{2}=\mathrm{G}\left[\frac{\phi+5}{2}\right] \frac{777424}{244755} .
\end{aligned}
$$

This expression does not diverge if $2<\phi$ and $-1+\frac{d}{\theta}<\phi$.

In the case of $\Gamma_{L} \sim \frac{\omega}{2}$ we find quite a different temperature dependence of $\chi_{3}^{\prime}(\omega, T)$ than for $\Gamma_{L}>3 \omega$ (Fig. 6). The 
frequency dependence is also different. $\chi_{3}^{\prime}(\omega, T)$ has singularity at $\omega \sim 2 \Gamma_{0}$, before this point we find the minima of $\chi_{3}^{\prime}$ depending on fixed temperature.

For $\Gamma_{L}<\frac{\omega}{2} \chi_{3}^{\prime}(\omega, T)$ has the minima on $T$ and temperatures of the minima are lower (approximately by ten times) than for $\Gamma_{L} \sim \frac{\omega}{2} \cdot \chi_{3}^{\prime}(\omega, T)$ as a function of $\omega$ has singularity at $\omega \sim 2 \Gamma_{0}$ (Fig. 7). The values of $\chi_{3}^{\prime}(\omega, T)$ at different fixed temperatures are approximately the same.

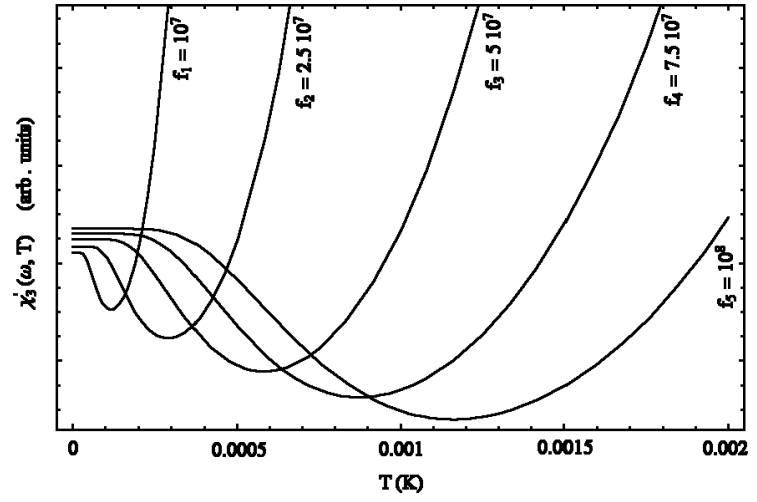

Fig. 6. The temperature dependence of the real part of the nonlinear dynamical susceptibility $\chi_{3}^{\prime}(\omega, T)$ at various frequencies $f$

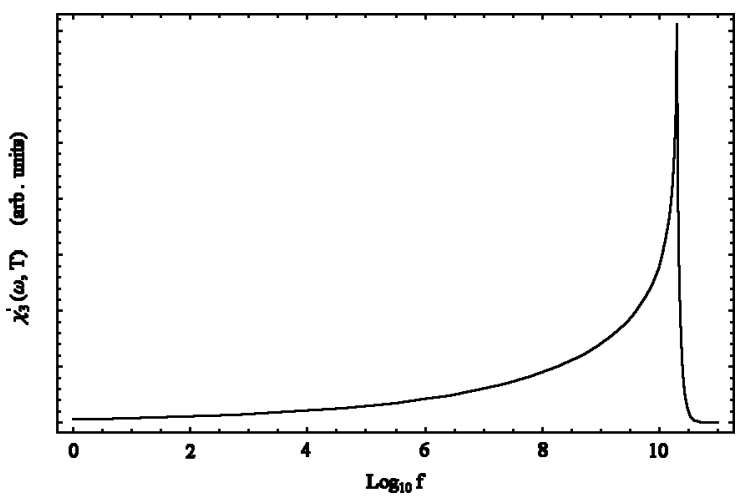

Fig. 7. The frequency dependence of the real part of the nonlinear dynamical susceptibility $\chi_{3}^{\prime}(\omega, T)$ versus $\log _{10} f$ at several fixed temperature
[1] Glassy dynamics and optimization, edited by Y. L. van Hemman, M. Morgenstern (Springer Verlag, Berlin Heidelberg, 1987).

[2] Spin-glasses and random fields, edited by A. P. Young (World Scientific, Singapore, 1997).

[3] T. Kopec, Phys. Rev. Lett. 79, 4266 (1997).

[4] D. S. Fisher, D. A. Huse, Phys. Rev. Lett. 56, 1601 (1986); Phys. Rev. B 36, 8937 (1987); Phys. Rev. B 38, 373, 386 (1988); D. S. Fisher, J. Appl. Phys. 61, 3672 (1987).

[5] A. Barrat, L. Berthier, preprint cond-mat/0102151 (2001); Y. G. Joh, R. Orbach. Phys. Rev. Lett. 77, 4648 (1996).

[6] M. J. Thill, D. A. Huse, Physica A 241, 321 (1995).

[7] H. Ishii, T. Yamamoto, J. Phys. C 18, 6225 (1985).

[8] N. Read, S. Sachdev, J. Ye, Phys. Rev. B 52, 384 (1995).

[9] H. Rieger, A. P. Young, Phys. Rev. Lett. 72, 4141 (1994).

[10] L. P. Lévi, A. T. Ogielski, Phys. Rev. Lett. 26, 3288 (1986).

[11] L. P. Lévi, Phys. Rev. B 38, 4963 (1988).

[12] T. Jonsson, K. Jonason, P. Jönsson, P. Nordblad, Phys. Rev. B 59, 8770 (1999)

[13] K. Gunnarsson, P. Svedlindh, P. Nordblad, L. Lundgren, H. Aruga, A. Ito, Phys. Rev. B 43, 8199 (1991); M. Hagiwara, T. Shimada, K. Miyoshi, M. Matsuura, J. Magn.
Magn. Mater. 177-181, 173 (1998)

[14] W. Wu, D. Bitko, T. F. Rosenbaum, G. Aeppli, Phys. Rev. Lett. 71, 1919 (1993); D. Bitko, T. F. Rosenbaum, G. Aeppli, Phys. Rev. Lett. 75, 1679 (1995).

[15] J. Mattsson, Phys. Rev. Lett. 75, 1678 (1995).

[16] G. Busiello, R. V. Saburova, Int. J. Mod. Phys. B 14, 1843 (2000); R. Saburova, G. P. Chugunova, G. Busiello, Phys. Met. Metallograph. 87, 509 (1999).

[17] R. Kubo, K. Tomita, J. Phys. Soc. Jpn. 9, 888 (1954); R. Kubo, J. Phys. Soc. Jpn. 12, 570 (1957).

[18] W. Bernard, H. B. Callen, Rev. Mod. Phys. 31, 1017 (1959); R. L. Peterson, Rev. Mod. Phys. 39, 69 (1967).

[19] W. T. Grandy, Jr. Foundation of statistical mechanics (D. Reidel Publ. Company, Dordrecht, Holland, 1988).

[20] W. Brenig, Statistical theory of heat (Springer Verlag, 1989).

[21] R. L. Stratonovich Nonlinear nonequilibrium thermodynamics. V. I (Springer Verlag, 1992); V. M. Fain, Kvantovaia Radiophizika (Quantum Radiophysics) (Izdatelstvo Sovietskoe Radio, Moskva, 1972).

[22] J. A. Mydosh, Spin Glasses: an experimental introduction (Taylor \& Francis, London, 1993).

[23] Tynneling systems in amorphous and crystalline solids, edited by P. Esquinazi (Springer Verlag, Heidelberg, 1998). 


\title{
ЛІНІЙНА ТА КУБІЧНА ДИНАМІЧНА СПРИЙНЯТЛИВІСТЬ У КВАНТОВОМУ СПІНОВОМУ СКЛІ
}

\author{
Дж. Бузіельйо ${ }^{1}$, Р. В. Сабурова ${ }^{2}$, В. Г. Сушкова ${ }^{2}$ \\ ${ }^{1}$ Фізичний відділ ім. Е. Р. Каяніельйо, Університет м. Салерно, 84081, Баронісі-Салерно \\ та відділ Національного інституту фізичного матеріллознавства, Салерно, Італіл \\ ${ }^{2}$ Фізичний факультет, Казансъкий енергетичний університет, \\ вул. Красносельська, 51, Казань, 420066, Росія
}

Досліджено низькотемпературну поведінку динамічної нелінійної (кубічної) сприйнятливости $\chi_{3}^{\prime}(\omega, T)$ у квантовому $d$-вимірному ізинговому спіновому склі з короткосяжною взаємодією поміж спінами. 3 пією метою застосовано квантову краплинну модель та квантовомеханічну нелінійну теорію відгуку. Отримано склоподібну поведінку краплинної динаміки. Виявлено сильну частотну залежність $\chi_{3}^{\prime}(\omega, T)$; температурну залежність помічено при дуже низьких температурах (квантовий режим). Нелінійний відпук залежний ві, темпу тунелювання краплі, що регулює величину квантових флюктуапій. Цей відгук сильно залежить від розподілу вільних енертій крапель та від середнього значення масштабу довжин крапель. Зазначено застосування результатів в експериментах над квантовими спіновими стеклами на зразок невпорядкованого дипольного квантового ізингового магнетика $\mathrm{LiHo}_{x} \mathrm{Y}_{1-x} \mathrm{~F}_{4}$ та псевдоспіну. 